\title{
Balanced domains and convexity
}

\author{
ARMEN EDIGARIAN
}

\begin{abstract}
For a quasi-balanced domain, we study holomorphic mappings $F: D \times D \rightarrow D$ such that $F(z, z)=z$ and $F(z, w)=F(w, z)$ for any $z, w \in D$. We show that in many cases the existence of such a function is equivalent to the convexity of the domain $D$.
\end{abstract}

1. Introduction. A fundamental theorem of Lempert states that on any convex domain, Caratheodory distance and Lempert function coincide (for definitions and results see [7], see also [6]). One of the main problems coming from Lempert's theorem is the fact that Carathéodory distance and Lempert function are biholomorphically invariant, however convexity is not. From the Riemann mapping theorem, any connected simply connected subdomain of $\mathbb{C}$ is biholomorphic to the unit disk $\mathbb{D}$ or to $\mathbb{C}$.

The main purpose of the paper is to study a condition which is biholomorphically invariant and is, in some cases, equivalent to convexity. Note that if $D \subset \mathbb{C}^{n}$ is a convex domain, then we have defined the following holomorphic mapping

$$
F: D \times D \ni(z, w) \mapsto \frac{z+w}{2} \in D .
$$

Now, let $D \subset \mathbb{C}^{n}$ be a domain. By $\mathcal{S}(D)$ we denote the set of all holomorphic mappings $F: D \times D \rightarrow D$ such that

$$
F(z, z)=z \quad \text { and } \quad F(z, w)=F(w, z) \quad \text { for any } z, w \in D .
$$

Hence, if $D$ is a convex domain, then $\mathcal{S}(D) \neq \varnothing$. Recall that a domain $D$ is balanced if for any $z \in D$ and any $\lambda \in \overline{\mathbb{D}}$ we have $\lambda z \in D$.

The paper was motivated by the following result.

Theorem 1. Let $D \Subset \mathbb{C}^{n}$ be a balanced pseudoconvex domain. Then the following conditions are equivalent: 
(1) $D$ is convex;

(2) $D$ is biholomorphic to a convex domain;

(3) $D$ can be exhausted by domains which are biholomorphic to convex domains, i.e., there exists a sequence $D_{1} \subset D_{2} \subset \cdots \subset D$ of domains biholomorphic to convex ones such that $D=\cup_{\nu \geq 1} D_{\nu}$.

(4) $\mathcal{S}(D) \neq \varnothing$.

The equivalence of conditions (1), (2), (3) in Theorem 1 is well-known (e.g. it follows from the results presented in [6]). The proof of Theorem 1 will be given in Section 4.

Note that the property $\mathcal{S}(D)=\varnothing$ is biholomorphically invariant (see below). So Theorem 1 shows that in some cases one can replace convexity by the existence of $F \in \mathcal{S}(D)$. We generalize this technique to the case of quasibalanced domains. We say that a domain $D \subset \mathbb{C}^{n}$ is $\left(\alpha_{1}, \ldots, \alpha_{n}\right)$-balanced if for any $z \in D$ and any $\lambda \in \overline{\mathbb{D}}$ we have $\left(\lambda^{\alpha_{1}} z_{1}, \ldots, \lambda^{\alpha_{n}} z_{n}\right) \in D$. Here, $\alpha_{1}, \ldots, \alpha_{n} \geq 0$ are integers. Note that a $(1, \ldots, 1)$-balanced domain is simply a balanced one. We say that $D$ is a quasi-balanced domain if it is $\left(\alpha_{1}, \ldots, \alpha_{n}\right)$ balanced for some positive integers $\alpha_{1}, \ldots, \alpha_{n}$.

For the sake of simplicity, we state our next result for a domain in $\mathbb{C}^{2}$ and for a (1,2)-balanced domain (a more general case is given in Theorem 5 below).

Theorem 2. Let $D \Subset \mathbb{C}^{2}$ be a $(1,2)$-balanced pseudoconvex domain. Then there exists a holomorphic mapping $F: D \times D \rightarrow D$ such that

(1) $F(z, z)=z$ for any $z \in D$;

(2) $F(z, w)=F(w, z)$ for any $z, w \in D$

if and only if there exists a $p \in \mathbb{C}$ such that for any $z, w \in D$ we have

$$
\left(\frac{z_{1}+w_{1}}{2}, \frac{z_{2}+w_{2}}{2}+p\left(z_{1}-w_{1}\right)^{2}\right) \in D .
$$

The open symmetrized bidisc $\mathbb{G}_{2}$ is the image of the bidisc $\mathbb{D}^{2}$ under the "symmetrization map" $\left(\lambda_{1}, \lambda_{2}\right) \rightarrow\left(\lambda_{1}+\lambda_{2}, \lambda_{1} \lambda_{2}\right)$. It is known that on $\mathbb{G}_{2}$ holomorphically invariant distances are equal (see [1-3]), $\mathbb{G}_{2}$ is not convex (see $[2]), \mathbb{G}_{2}$ cannot be exhausted by domains which are biholomorphic to convex domains (see [4]), $\mathbb{G}_{2}$ can be exhausted by domains which are strictly linearly convex (see [10]). Note that $\mathbb{G}_{2}$ is a $(1,2)$-balanced pseudoconvex domain.

As a corollary of Theorem 2, we have

Corollary 3. $\mathcal{S}\left(\mathbb{G}_{2}\right)=\varnothing$.

The proof of Corollary 3 will be given in Section 4. The following question is posed in [9].

Question. Does there exist a domain $G$ in some $\mathbb{C}^{n}$ so that $\mathbb{G}_{2} \times G$ is biholomorphic to a convex domain?

Note that if there exist domains $G, D$, where $D$ is convex, and a biholomorphic mapping $f: \mathbb{G}_{2} \times G \rightarrow D$, then we can fix any point $w_{0} \in G$ and consider a mapping $F\left(z_{1}, z_{2}\right)=\operatorname{proj}_{1} \circ g\left(\frac{f\left(z_{1}, w_{0}\right)+f\left(z_{2}, w_{0}\right)}{2}\right)$, for $z_{1}, z_{2} \in \mathbb{G}_{2}$, where $g=f^{-1}$ and $\operatorname{proj}_{1}: \mathbb{G}_{2} \times G \rightarrow \mathbb{G}_{2}$ is the projection onto the first 
variable. The function $F$ cannot exist. So, the answer to the above mentioned question is negative. It is a generalization of Theorem 2 in [9].

2. Holomorphic functions between balanced domains. From the Taylor series expansion, it is easy to get the following useful result.

Lemma 4. Let $D \subset \mathbb{C}^{n}$ be a domain containing 0 , and let $\Phi: D \rightarrow \mathbb{C}$ be a holomorphic function. Assume that $\alpha_{1}, \ldots, \alpha_{n}, \beta$ are positive integers. For any $z \in D$ we put

$$
\varphi(\lambda)=\frac{1}{\lambda^{\beta}} \Phi\left(\lambda^{\alpha_{1}} z_{1}, \ldots, \lambda^{\alpha_{n}} z_{n}\right) .
$$

Note that $\varphi$ is holomorphic on a punctured neighborhood of zero. Then $\varphi(0)$ exists for any $z \in D$ if and only if $c_{\gamma}=0$ for any $\gamma$ such that $\alpha \cdot \gamma<\beta$. Here, $\Phi(z)=\sum_{\gamma \in \mathbb{Z}_{+}^{n}} c_{\gamma} z^{\gamma}$ is a Taylor series of $\Phi$. Moreover, then

$$
\varphi(0)=\sum_{\gamma \in \mathbb{Z}_{+}^{n}, \alpha \cdot \gamma=\beta} c_{\gamma} z^{\gamma} .
$$

Let us write more explicitly the case when $\alpha_{1}, \ldots, \alpha_{n}, \beta \in\{1,2\}$. Put $S_{\ell}(D)=\left\{j \in\{1, \ldots, n\}: \alpha_{j}=\ell\right\}$ for $\ell=1, \ldots, n$. Then we can write the above Lemma in the following way.

- For $\beta=1$ we have $\varphi(0)$ exists if and only if $\Phi(0)=0$. Then $\varphi(0)=$ $\sum_{j \in S_{1}} \frac{\partial \Phi}{\partial z_{j}}(0) z_{j}$.

- For $\beta=2$ we have $\varphi(0)$ exists if and only if $\Phi(0)=0$ and $\frac{\partial \Phi}{\partial z_{j}}(0)=0$ for $j \in S_{1}$. Then

$$
\varphi(0)=\sum_{j \in S_{2}} \frac{\partial \Phi}{\partial z_{j}}(0) z_{j}+\frac{1}{2} \sum_{j, k \in S_{1}} \frac{\partial^{2} \Phi}{\partial z_{j} \partial z_{k}}(0) z_{j} z_{k} .
$$

As an application of the above Lemma, we have the following Cartan-type result for some quasi-balanced domains.

Theorem 5. Let $D \subset \mathbb{C}^{n}$ be $\left(\alpha_{1}, \ldots, \alpha_{n}\right)$-balanced, and let $G \subset \mathbb{C}^{m}$ be a $\left(\beta_{1}, \ldots, \beta_{m}\right)$-balanced, where $\alpha_{1}, \ldots, \alpha_{n}, \beta_{1}, \ldots, \beta_{m} \in\{1,2\}$. Assume that $G$ is pseudoconvex and that $F: D \rightarrow G$ is a holomorphic mapping such that $F(0)=0$ and $\frac{\partial F_{\ell}}{\partial z_{j}}(0)=0$ for all $j \in S_{1}(D)$ and all $\ell \in S_{2}(G)$. Then $\widetilde{F}(D) \subset G$, where

$\widetilde{F}_{\ell}(z)=\left\{\begin{array}{ll}\sum_{j \in S_{1}(D)} \frac{\partial F_{\ell}}{\partial z_{j}}(0) z_{j}, & \text { when } \ell \in S_{1}(G) \\ \sum_{j \in S_{2}(D)} \frac{\partial F_{\ell}}{\partial z_{j}}(0) z_{j}+\frac{1}{2} \sum_{j, k \in S_{1}(D)} \frac{\partial^{2} F_{\ell}}{\partial z_{j} \partial z_{k}}(0) z_{j} z_{k}, & \text { when } \ell \in S_{2}(G)\end{array}\right.$.

Before we go into the proof, recall the equivalent condition for pseudoconvexity of quasi-balanced domains. Let $D$ be a $\left(\alpha_{1}, \ldots, \alpha_{n}\right)$-balanced domain. We put $\pi_{\lambda}(z)=\left(\lambda^{\alpha_{1}} z_{1}, \ldots, \lambda^{\alpha_{n}} z_{n}\right)$ and

$$
h_{D}(z)=\inf \left\{t>0: \pi_{1 / t}(z) \in D\right\}, \quad z \in \mathbb{C}^{n} .
$$

Then $D$ is pseudoconvex if and only if $\log h_{D} \in \operatorname{PSH}\left(\mathbb{C}^{n}\right)$ (see e.g. [8]). Note that $h_{D}\left(\pi_{\lambda}(z)\right)=|\lambda| h_{D}(z)$ for any $\lambda \in \mathbb{C}$ and $z \in \mathbb{C}^{n}$. Moreover, $D=\{z \in$ $\left.\mathbb{C}^{n}: h_{D}(z)<1\right\}$. 
Note that if $D$ is quasi-balanced and pseudoconvex, then for any holomorphic mapping $F: G \rightarrow \bar{D}$ we have $F(G) \subset D$ or $F(G) \subset \partial D$.

Proof of Theorem 5 For a fixed $z \in D$, consider a mapping

$$
\psi(\lambda)=\left(\frac{F_{1}\left(\lambda^{\alpha_{1}} z_{1}, \ldots, \lambda^{\alpha_{n}} z_{n}\right)}{\lambda^{\beta_{1}}}, \ldots, \frac{F_{m}\left(\lambda^{\alpha_{1}} z_{1}, \ldots, \lambda^{\alpha_{n}} z_{n}\right)}{\lambda^{\beta_{m}}}\right) .
$$

Note that $\psi$ extends holomorphically to 0 . Moreover, there exists $\rho \in(0,1)$ such that $h_{G}(\psi(\lambda)) \leq \rho$ for any $\lambda \in \partial \mathbb{D}$. Hence, $h_{G}(\psi(0)) \leq \max _{\lambda \in \partial \mathbb{D}} h_{G}$ $(\psi(\lambda)) \leq \rho$ and, therefore, $\psi(0) \in G$.

For balanced domains (i.e., $\alpha_{1}=\cdots=\alpha_{n}=\beta_{1}=\cdots=\beta_{m}=1$ ), from Theorem 5 we get:

Corollary 6. Let $D \subset \mathbb{C}^{n}, G \subset \mathbb{C}^{m}$ be balanced domains. Assume that $G$ is pseudoconvex. If $F: D \rightarrow G$ is a holomorphic mapping such that $F(0)=0$, then $F^{\prime}(0) z \in G$ for any $z \in D$.

3. Study of the condition $\mathcal{S}(\boldsymbol{D}) \neq \varnothing$. The following result (we state it as a theorem, although it is a simple remark) shows why we are interested in exhaustion by domains biholomorphic to a convex domain.

Theorem 7. Let $G \subset \mathbb{C}^{n}$ be a domain. Suppose that there exist a sequence of domains $G_{\nu} \subset G, D_{\nu} \subset \mathbb{C}^{n_{\nu}}, \nu \in \mathbb{N}$, holomorphic mappings $\varphi_{\nu}: G_{\nu} \rightarrow D_{\nu}$, $\psi_{\nu}: D_{\nu} \rightarrow G$ such that

- on $D_{\nu}$ holomorphic distances coincide;

- $G_{1} \subset G_{2} \subset \ldots, \cup_{\nu \geq 1} G_{\nu}=G$;

- $\psi_{\nu} \circ \varphi_{\nu}=\operatorname{id}_{G_{\nu}}$.

Then on $G$ holomorphic distances coincide.

In particular, if domains $D_{\nu}$ are convex, then on $G$ holomorphic distances coincide. We give a simple proof.

Proof of Theorem 7 Fix $z_{1}, z_{2} \in G$. Then

$$
c_{G_{\nu}}\left(z_{1}, z_{2}\right) \geq \widetilde{k}_{D_{\nu}}\left(\varphi\left(z_{1}\right), \varphi\left(z_{2}\right)\right) \geq \widetilde{k}_{G}\left(z_{1}, z_{2}\right) .
$$

But $c_{G_{\nu}}\left(z_{1}, z_{2}\right) \rightarrow c_{G}\left(z_{1}, z_{2}\right)$ when $\nu \rightarrow \infty$ (see e.g. [6]).

Now we study invariance of the property $\mathcal{S}(D) \neq \varnothing$ under biholomorphic mappings and exhaustion of domains.

Proposition 8. Let $D_{1} \subset D_{2} \subset \cdots \subset D$ be domains such that $D=\cup_{\nu \geq 1} D_{\nu}$. Assume that for any $\nu \geq 1$ there exists a holomorphic mapping $F_{\nu}: D_{\nu} \times D_{\nu} \rightarrow$ $\overline{D_{\nu}}$ such that $F_{\nu}(z, z)=z$ and $F_{\nu}(z, w)=F_{\nu}(w, z)$ for any $z, w \in D_{\nu}$. Then there exists a holomorphic mapping $F: D \times D \rightarrow \bar{D}$ such that $F(z, z)=z$ and $F(z, w)=F(w, z)$ for any $z, w \in D$.

Proof. Follows easily from Montel's theorem.

Proposition 9. Let $D, G \subset \mathbb{C}^{n}$ be domains, and let $\Phi: D \rightarrow G$ be a biholomorphic mapping. Assume that there exists a holomorphic mapping $F: D \times D \rightarrow D$ such that $F(z, z)=z$ and $F(z, w)=F(w, z)$ for any $z, w \in D$. Then there 
exists a holomorphic mapping $\widetilde{F}: G \times G \rightarrow G$ such that $\widetilde{F}(z, z)=z$ and $\widetilde{F}(z, w)=\widetilde{F}(w, z)$ for any $z, w \in G$. $\varnothing$.

In particular, if $G$ is biholomorphic to a convex domain, then the set $\mathcal{S}(G) \neq$

Proof. Just put $\widetilde{F}(z, w)=\Phi \circ F\left(\Phi^{-1}(z), \Phi^{-1}(w)\right)$.

Using Corollary 3 and similar arguments as above, we have the following result.

Corollary 10. There does not exist a sequence of domains $G_{\nu} \subset \mathbb{C}^{2}, \nu \geq 1$, such that:

(1) $G_{1} \subset G_{2} \subset \cdots \subset \mathbb{G}_{2}$;

(2) $\cup_{\nu \geq 1} G_{\nu}=\mathbb{G}_{2}$;

(3) there exist holomorphic mappings $\varphi_{\nu}: G_{\nu} \rightarrow D_{\nu}, \psi_{\nu}: D_{\nu} \rightarrow \mathbb{C}^{2}$ such that $\psi_{\nu} \circ \varphi_{\nu} \rightarrow \mathrm{id}_{\mathbb{G}_{2}}$ pointwise, where $D_{\nu} \subset \mathbb{C}^{m_{\nu}}$ is a convex domain, $m_{\nu} \in \mathbb{N}$.

(4) $\psi_{\nu}$ are uniformly bounded, i.e., there exists a constant $C>0$ such that $\sup _{w \in D_{\nu}}\left|\psi_{\nu}(w)\right| \leq C, \nu \geq 1$.

Proof. Assume that such a sequence exists. Put $F_{\nu}\left(z_{1}, z_{2}\right)=\psi_{\nu}\left(\frac{\varphi_{\nu}\left(z_{1}\right)+\varphi_{\nu}\left(z_{2}\right)}{2}\right)$. Then $F_{\nu}: G_{\nu} \times G_{\nu} \rightarrow \mathbb{C}_{2}$ is a holomorphic mapping such that $F_{\nu}(z, w)=$ $F_{\nu}(w, z)$. Moreover, $F_{\nu}(z, z) \rightarrow z$ for any $z \in \mathbb{G}_{2}$. Since $\left\{F_{\nu}\right\}$ is uniformly bounded, from Montel's theorem there exists a subsequence $n_{k}$ such that $F_{n_{k}} \rightarrow F$ locally uniformly. But then $F: \mathbb{G}_{2} \times \mathbb{G}_{2} \rightarrow \overline{\mathbb{G}}_{2}$ and $F(z, z)=z$ and $F(z, w)=F(w, z)$ for any $z, w \in \mathbb{G}_{2}$. This is a contradiction.

Remark 11. Actually, similar arguments show that one can take domains $D_{\nu} \subset$ $H_{\nu}$, where $H_{\nu}$ is a complex linear topological space.

Remark 12. Similar ideas can be used to study biholomorphicity to convex domains of the tetrablock (cf. [5]) and of higher dimensional symmetrized polydiscs (cf. [9]).

4. Proofs of Theorem 1 and Corollary 3. Assume that $D \subset \mathbb{C}^{n}$ is a domain and that $F \in \mathcal{S}(D)$. Then for any $z, w \in D$ and $j, k, \ell=1,2, \ldots, n$, we have

$$
\begin{aligned}
\frac{\partial F_{\ell}}{\partial z_{j}}(z, z) & =0, \quad \text { for any } j \neq \ell, \\
\frac{\partial F_{\ell}}{\partial z_{\ell}}(z, z) & =\frac{1}{2}, \\
\frac{\partial^{2} F_{\ell}}{\partial z_{j} \partial z_{k}}(z, z) & =\frac{\partial^{2} F_{\ell}}{\partial w_{j} \partial w_{k}}(z, z)=-\frac{\partial^{2} F_{\ell}}{\partial z_{j} \partial w_{k}}(z, z) .
\end{aligned}
$$

As a corollary of Theorem 5, we get:

Corollary 13. Let $D \subset \mathbb{C}^{n}$ be an $\left(\alpha_{1}, \ldots, \alpha_{n}\right)$-balanced pseudoconvex domain such that $\alpha_{1}, \ldots, \alpha_{n} \in\{1,2\}$. Assume that $F \in \mathcal{S}(D)$. Then $\widetilde{F} \in \mathcal{S}(D)$, where $\widetilde{F}_{\ell}(z, w)$

$$
=\left\{\begin{array}{l}
\frac{z_{\ell}+w_{\ell}}{2} \quad \text { when } \ell \in S_{1}(D), \\
\frac{z_{\ell}+w_{\ell}}{2}+\frac{1}{2} \sum_{j, k \in S_{1}(D)} \frac{\partial^{2} F_{\ell}}{\partial z_{j} \partial z_{k}}(0,0)\left(z_{j}-w_{j}\right)\left(z_{k}-w_{k}\right), \quad \text { when } \ell \in S_{2}(D)
\end{array} .\right.
$$


In particular, if $D \subset \mathbb{C}^{2}$ is a $(1,2)$-balanced pseudoconvex domain and if $F \in \mathcal{S}(D)$, then $\widetilde{F} \in \mathcal{S}(D)$, where

$$
\widetilde{F}(z, w)=\left(\frac{z_{1}+w_{1}}{2}, \frac{z_{2}+w_{2}}{2}+\frac{1}{2} \cdot \frac{\partial^{2} F_{2}}{\partial z_{1}^{2}}(0,0)\left(z_{1}-w_{1}\right)^{2}\right) .
$$

Let us go to the Proof of Theorem 1.

Proof. (1) $\Longrightarrow(2) \Longrightarrow(3)$ are clear. Let us show the implication $(3) \Longrightarrow$ (4). Suppose that $\Phi_{\nu}: D_{\nu} \rightarrow G_{\nu}$ is a biholomorphic mapping and that $G_{\nu}$ is convex. We put $F_{\nu}(z, w)=\Phi_{\nu}^{-1}\left(\frac{\Phi_{\nu}(z)+\Phi_{\nu}(w)}{2}\right)$. Note that $F_{\nu}: D_{\nu} \times D_{\nu} \rightarrow D_{\nu}$ is a holomorphic mapping such that $F_{\nu}(z, z)=z$ and $F_{\nu}(z, w)=F_{\nu}(w, z)$ for any $z, w \in D_{\nu}$. By Montel's theorem there exists a subsequence $F_{\nu_{k}} \rightarrow F$, where $F: D \times D \rightarrow D$ such that $F(z, z)=z$ and $F(z, w)=F(w, z)$.

$(4) \Longrightarrow(1)$. Since here $S_{1}(D)=\{1, \ldots, n\}$, the proof follows from Corollary 13 .

Proof of Corollary 3 Assume that such a function exists. Then by Theorem 2 there exists a $p \in \mathbb{C}$ such that

$$
\left(\frac{z_{1}+w_{1}}{2}, \frac{z_{2}+w_{2}}{2}+p\left(z_{1}-w_{1}\right)^{2}\right) \in \mathbb{G}_{2}
$$

for any $\left(z_{1}, z_{2}\right),\left(w_{1}, w_{2}\right) \in \mathbb{G}_{2}$.

Consider $(\zeta-\eta,-\eta \zeta),(\zeta+\eta, \eta \zeta) \in \mathbb{G}_{2}$, where $\zeta, \eta \in \mathbb{D}$. Hence,

$$
\left(\zeta, 4 p \eta^{2}\right) \in \mathbb{G}_{2} \text {. }
$$

Therefore, $(t,-4|p| t) \in \mathbb{G}_{2}$ for any $t \in(0,1)$. Note that $\left(z_{1}, z_{2}\right) \in \mathbb{G}_{2}$ if and only if the equation $x^{2}-z_{1} x+z_{2}=0$ has solutions in $\mathbb{D}$. If $p \neq 0$, then we may take any $t \in\left(\frac{1}{1+4|p|}, 1\right)$ and get that the equation $x^{2}-t x-4|p| t=0$ has a solution $x=\frac{t+\sqrt{t^{2}+16 t|p|}}{2}>1$. Hence, $p=0$. So, $\mathbb{G}_{2}$ is convex, but it is a contradiction (take e.g. $\left(2 t, t^{2}\right),\left(2 i t,-t^{2}\right) \in \mathbb{G}_{2}$, where $t \in(1 / \sqrt{2}, 1)$, but $\left.(t(1+i), 0) \notin \mathbb{G}_{2}\right)$.

Acknowledgements. The author thanks Marek Jarnicki, Peter Pflug, and Włodzimierz Zwonek for helpful remarks. The author thanks also the anonymous referee for careful reading and precious remarks.

Open Access. This article is distributed under the terms of the Creative Commons Attribution License which permits any use, distribution, and reproduction in any medium, provided the original author(s) and the source are credited.

\section{References}

[1] J. Agler And N.J. Young, The hyperbolic geometry of the symmetrized bidisc, J. Geom. Anal. 14 (2004), 375-403.

[2] C. Costara, The symmetrized bidisc and Lempert's theorem, Bull. London Math. Soc., 36 (2004), 656-662.

[3] C. Costara, Dissertation, Université Laval (2004). 
[4] A. Edigarian, A note on Costara's paper, Ann. Polon. Math. 83 (2004), 189191.

[5] A. Edigarian And W. Zwonek, Schwarz lemma for the tetrablock, Bull. London Math. Soc. 41 (2009), 506-514.

[6] M. Jarnicki And P. Pflug, Invariant Distances and Metrics in Complex Analysis, Walter de Gruyter, 1993.

[7] L. Lempert, La métrique de Kobayashi et la représentation des domaines sur la boule, Bull. Soc. Math. France 109 (1981), 427-474.

[8] N. Nikolov, The symmetrized polydisk cannot be exhausted by domains biholomorphic to convex domains, Ann. Pol. Math. 88 (2006), 279-283.

[9] N. Nikolov, P. Pflug, And W. Zwonek, The Lempert function of the symmetrized polydisc in higher dimensions is not a distance, Proc. A.M.S. 135 (2007), 2921-2928.

[10] P. Pflug And W. Zwonek, Exhausting domains of the symmetrized bidisc, Ark. Mat. 50 (2012), 397-402.

\section{ARMEn EdigARIAN}

Wydział Matematyki i Informatyki, Uniwersytet Jagielloński, ul. Prof. St. Łojasiewicza 6, 30-348 Kraków, Poland

e-mail: armen.edigarian@uj.edu.pl

Received: 23 January 2013 